Pacific Journal of Mathematics

TWO PRIMARY FACTOR INEQUALITIES 


\section{TWO PRIMARY FACTOR INEQUALITIES}

\section{J. H. E. COHN}

In the theory of integral functions, the expressions

$$
E(z, p)=(1-z) \exp \left\{\sum_{1}^{p} \frac{z^{r}}{r}\right\}, p=1,2, \cdots
$$

called primary factors, are of some importance, and it is of interest to find upper bounds for $|E(z, p)|$. Clearly $E(z, p)=0$ only for $z=1$, and so for other values, define $f(z, p)=$ $\log |E(z, p)|$. It is known that for suitable constants $a_{p}, b_{p}$ the inequalities

$$
\begin{aligned}
& f(z, p) \leqq a_{p}|z|^{p},|z| \geqq 1, z \neq 1 \\
& f(z, p) \leqq b_{p}|z|^{p+1},|z| \leqq 1, z \neq 1
\end{aligned}
$$

are satisfied; for instance Hille has shown that one may take $a_{p}=1+\sum_{1}^{p} 1 / r \leqq 2+\log p$ and $b_{p}=1$.

In this paper, the smallest values of both $a_{p}$ and $b_{p}$ are determined, the latter in closed form.

Throughout, we shall write $z=\rho e^{i \theta}$, where without loss of generality $\rho \geqq 0,0 \leqq \theta \leqq \pi$. Then

$$
f(z, p)=\frac{1}{2} \log \left(1-2 \rho \cos \theta+\rho^{2}\right)+\sum_{1}^{p} \frac{\rho^{r}}{r} \cos r \theta
$$

Also, using the Taylor series for $\log (1-z)$ gives from (1)

$$
f(z, p)=-\rho^{p+1} \sum_{0}^{\infty} \frac{\rho^{r}}{p+r+1} \cos (p+r+1) \theta,
$$

provided $\rho<1$. A further expression is obtained by writing $\log E(z, p)$ as an integral of its derivative and taking real parts, to give

$$
f(z, p)=\int_{0}^{\rho} \frac{t \cos p \theta-\cos (p+1) \theta}{1-2 t \cos \theta+t^{2}} t^{p} d t
$$

provided $\theta \neq 0$ or $\rho<1$.

The problem considered in this paper is the determination of the maxima of the functions

$$
g(z, p)=\rho^{-p} f(z, p) \text { for } \rho \geqq 1
$$

and

$$
h(z, p)=\rho^{-p-1} f(z, p) \text { for } \rho \leqq 1 \text {, }
$$


and to show where these occur.

1. Summary of results. Henceforth we use $a_{p}$ and $b_{p}$ to denote the smallest constants for which (2) and (3) hold. We shall show that both $a_{p}$ and $b_{p}$ are monotone decreasing functions of $p$. The value of $a_{1}$ is given by $a_{1}=\log (\rho-1)$ where $\rho$ is the solution of the transcendental equation $(\rho-1) \log (\rho-1)=\rho, \rho>1$ and the maximum occurs at $z=\rho$. Also $a_{2}=1$, the maximum occuring at $z=2$, and $a_{\infty}$ is given by the common value of $x^{-1}$ and

$$
e^{-x}\left(y+\int_{0}^{1} \frac{e^{s}-1}{s} d s+\int_{1}^{x} \frac{e^{s}}{s} d s\right)
$$

for the unique value of $x$ which makes these expressions equal, $\gamma$ denoting Euler's constant. For each $p \geqq 2$, the maximum occurs at a point $z$ on the real axis which satisfies $1<z \leqq 2$.

The $z$ maximizing $b_{p}$ occur on $|z|=1$, with $\theta=\pi /(2 p+1), p=1$, $2,3, \cdots$. For $p>1$ the maximum is unique, but for $p=1$ it is attained at every point of the arc $|z-1|=1,|z| \leqq 1$. We derive the explicit bounds

$$
\frac{1}{2} \geqq b_{p}>\log \pi / 2+\gamma-\int_{0}^{\pi / 2} \frac{1-\cos x}{x} d x,
$$

and both bounds are sharp. We also have an explicit formula

$$
b_{p}=\log \left(2 \sin \frac{1}{2} \theta\right)+\sum_{1}^{p} \frac{1}{r} \cos r \theta,
$$

where $\theta=\pi /(2 p+1)$. In particular these results give

$$
1.2785 \geqq a_{p}>0.7423, \frac{1}{2} \geqq b_{p}>0.4719 \text {. }
$$

Since $a_{2}=1$ we have therefore

$$
\log |E(z, p)| \leqq \min \left(|z|^{p},|z|^{p+1}\right), p=2,3, \cdots,
$$

and this is sharp.

The numerical values of $a_{p}$ and $b_{p}$ are as follows.

$\begin{array}{ccc}p & a_{p} & b_{p} \\ 1 & 1.2785 & 0.5000 \\ 2 & 1.0000 & 0.4823 \\ 3 & 0.9123 & 0.4771 \\ 4 & 0.8691 & 0.4752 \\ 5 & 0.8435 & 0.4741 \\ \infty & 0.7423 & 0.4719\end{array}$


2. Preliminaries. It is clear that for (2) and (3) to hold, both $a_{p}$ and $b_{p}$ must be positive, since for example $z=2$ and $z=\varepsilon \exp i \pi /(p+1)$ with $\varepsilon$ sufficiently small give positive values of $f(z, p)$. We see therefore that not only the point $z=1$, but also a neighbourhood of this point can be excluded from the discussion. We find by elementary means that

(8) $\frac{\partial g}{\partial \theta}=\frac{\rho\{\sin (p+1) \theta-\rho \sin p \theta\}}{1-2 \rho \cos \theta+\rho^{2}}$

(9) $\frac{\partial^{2} g}{\partial \theta^{2}}=\frac{\partial\{(p+1) \cos (p+1) \theta-p \rho \cos p \theta\}}{1-2 \rho \cos \theta+\rho^{2}}-\frac{2 \rho \sin \theta}{1-2 \rho \cos \theta+\rho^{2}} \frac{\partial g}{\partial \theta}$

(10) $\frac{\partial g}{\partial \rho}=-\frac{p g}{\rho}+\frac{\rho \cos p \theta-\cos (p+1) \theta}{1-2 \rho \cos \theta+\rho^{2}}$

$$
\begin{aligned}
\frac{\partial h}{\partial \theta} & =\frac{\sin (p+1) \theta-\rho \sin p \theta}{1-2 \rho \cos \theta+\rho^{2}} \\
\frac{\partial^{2} h}{\partial \theta^{2}} & =\frac{(p+1) \cos (p+1) \theta-p \rho \cos \theta}{1-2 \rho \cos \theta+\rho^{2}}-\frac{2 \rho \sin \theta}{1-2 \rho \cos \theta+\rho^{2}} \frac{\partial h}{\partial \theta} \\
\frac{\partial h}{\partial \rho} & =-\frac{(p+1) h}{\rho}+\frac{\rho \cos p \theta-\cos (p+1) \theta}{\rho\left(1-2 \rho \cos \theta+\rho^{2}\right)}
\end{aligned}
$$

3. The case $\rho=1$. We consider first the unit circle on which of course $f, g$ and $h$ coincide, with $0 \leqq \theta \leqq \pi$. Then by (8) we find that $\partial f / \partial \theta=1 / 2 \cos (p+(1 / 2)) \theta \operatorname{cosec}(1 / 2) \theta$, and so local maxima occur at $\theta=\beta, 5 \beta, 9 \beta, \cdots$ where $\beta=\pi /(2 p+1)$. We shall show that $f(\beta)>$ $f(5 \beta)>f(9 \beta)>\cdots$ and hence that $f(\beta)$ is the largest value taken by $f(z, p)$ on $|z|=1$. For, let $n \geqq 0$ with $(4 n+5) \beta \leqq \pi$. Then

$$
\begin{aligned}
& f((4 n+5) \beta)-f((4 n+1) \beta)=\int_{(4 n+1) \beta}^{(4 n+5) \beta} f^{\prime}(\theta) d \theta \\
= & \frac{1}{2} \int_{(4 n+1) \beta}^{(4 n+3) \beta} \cos \frac{\pi \theta}{2 \beta} \operatorname{cosec} \frac{1}{2} \theta d \theta+\frac{1}{2} \int_{(4 n+3) \beta}^{(4 n+5) \beta} \cos \frac{\pi \theta}{2 \beta} \operatorname{cosec} \frac{1}{2} \theta d \theta \\
= & \frac{-\beta}{2 \pi} \int_{0}^{2 \pi} \frac{\sin \frac{1}{2} \phi}{\sin \left(4 n+3-\frac{\phi}{\pi}\right) \frac{\beta}{2}} d \phi+\frac{\beta}{2 \pi} \int_{0}^{2 \pi} \frac{\sin \frac{1}{2} \phi}{\sin \left(4 n+3+\frac{\phi}{\pi}\right) \frac{\beta}{2}} d \phi \\
< & 0,
\end{aligned}
$$

where we have substituted $\phi=(4 n+3) \pi-\pi \theta / \beta$ in the first integral, and $\phi=-(4 n+3) \pi+\pi \theta / \beta$ in the second.

Thus we obtain in view of (4), that for $|z|=1$,

$$
f(z, p) \leqq \sigma_{p}=\log \left(2 \sin \frac{\pi}{4 p+2}\right)+\sum_{1}^{p} \frac{1}{r} \cos \frac{r \pi}{2 p+1} .
$$


We now consider $\sigma_{p}$, and prove first that $\sigma_{p}>\sigma_{p+1}$. Define $\delta$ by $\pi=2(2 p+1)(2 p+3) \delta$. Then

$$
\begin{aligned}
\sigma_{p}-\sigma_{p+1}= & \log \frac{\sin (2 p+3) \delta}{\sin (2 p+1) \delta}+\sum_{1}^{p} \frac{1}{r}\{\cos 2(2 p+3) r \delta-\cos 2(2 p+1) r \delta\} \\
& -\frac{1}{p+1} \cos 2(2 p+1)(p+1) \delta \\
& =\lambda(\delta), \text { say. }
\end{aligned}
$$

Thus if $\lambda(\phi)$ is defined for $0<\phi \leqq \delta$ by the same formula with $\delta$ replaced by $\phi$, we find that as $\phi \rightarrow 0$,

$$
\lambda(\phi) \rightarrow \log \frac{2 p+3}{2 p+1}-\frac{1}{p+1}>0 .
$$

Also

$$
\begin{aligned}
\lambda^{\prime}(\phi) & =\{(2 p+3) \operatorname{cosec}(2 p+3) \phi-(2 p+1) \operatorname{cosec}(2 p+1) \phi\} \cos \frac{\pi \phi}{2 \delta} \\
& >0,
\end{aligned}
$$

since $x \operatorname{cosec} x$ is strictly increasing in $(0, \pi / 2)$.

Thus $\lambda(\delta)>0$, and so

$$
\sigma_{p}>\sigma_{p+1}
$$

Also as $p \rightarrow \infty$ we find that

$$
\begin{aligned}
\sigma_{p}= & \log \left(2 \sin \frac{\pi}{4 p+2}\right)+\sum_{1}^{p} \frac{1}{r} \cos \frac{r \pi}{2 p+1} \\
= & \log \frac{\pi}{2 p+1}+o(1)+\sum_{1}^{p} \frac{1}{r}+\sum_{1}^{p} \frac{1}{r}\left\{\cos \frac{r \pi}{2 p+1}-1\right\} \\
= & \log \pi / 2+\left\{\sum_{1}^{p} \frac{1}{r}-\log p\right\}-\int_{0}^{\pi / 2} \frac{1-\cos x}{x} d x+o(1) \\
& \rightarrow \log \frac{1}{2} \pi+\gamma-\int_{0}^{\pi / 2} \frac{1-\cos x}{x} d x=0.4719 .
\end{aligned}
$$

Thus we find, since $\sigma_{1}=1 / 2$, that for all $p$

$$
\frac{1}{2} \geqq \sigma_{p}>0.4719 \text {. }
$$

4. The case $\rho \leqq 1$. For $\rho \leqq 1$, we consider first $p=1$, where the situation is slightly different from the remaining values of $p$. Using (11) we see that if $\rho \neq 1$, then for fixed $\rho, h$ has turning values, regarded as a function of $\theta$, only for $\theta=0, \theta=\pi$ and $2 \cos \theta=\rho$. Using (12) we find that both $\theta=0$ and $\theta=\pi$ give minima, and so for each $\rho \in(0,1)$ we find that 


$$
h(z, 1) \leqq \rho^{-2}\left(\frac{1}{2} \log 1+\frac{1}{2} \rho^{2}\right)=\frac{1}{2},
$$

with equality if and only if $2 \cos \theta=\rho$. Thus we have $b_{1}=1 / 2$ with equality attained at every point of the arc $|z-1|=1,|z| \leqq 1$.

For $p \geqq 2$, the situation is quite different. Clearly whatever $b_{p}$ with turn out to be, there will be equality in (3) for $z=0$. But for $0<\rho<2 / 7$, we find using (5) and (7) that

$$
\begin{aligned}
h(z, p)= & -\sum_{0}^{\infty} \frac{\rho^{r}}{p+r+1} \cos (p+r+1) \theta \\
& <\frac{1}{3} \sum_{0}^{\infty}\left(\frac{2}{7}\right)^{r}=\frac{7}{15}<\sigma_{p}, \text { by }(16) .
\end{aligned}
$$

Thus the maximum of $h(z, p)$ occurs in the closed annulus $2 / 7 \leqq$ $\rho \leqq 1$.

Again consider a fixed value of $p<1$. By (11) the greatest value of $h$, regarded as a function of $\theta$, occurs at a solution of $\sin (p+$ 1) $\theta=\rho \sin p \theta . \quad \theta=0$ is impossible since then $\partial^{2} h / \partial \theta^{2}>0$ by (12) and $\theta=\pi$ can be neglected since then by (5) and (7) we get

$$
h(-\rho, p)=\sum_{0}^{\infty} \frac{\rho^{r}}{p+r+1}(-1)^{p+r} \leqq \frac{1}{p+1} \leqq \frac{1}{3}<\sigma_{p} .
$$

A glance at the sketch of $y=\sin (p+1) x / \sin p x$ for $x \in(0, \pi)$, shown in Figure 1, reveals that there are precisely $p$ other values of $\theta$ to consider, since it is readily shown that each branch of the curve is monotone strictly decreasing. Again we consider the sign of $\partial^{2} h / \partial \theta^{2}$. Since $\rho<1$ we find that for given $\rho$, the intersection of $y=\rho$ with the $r$ th. branch of the curve satisfies

$$
\frac{2 r-1}{2 p+1} \pi<\theta<\frac{r \pi}{p+1}
$$

whence $p \theta \in((r-1) \pi, r \pi)$ and $(p+1) \theta \in((r-(1 / 2)) \pi, r \pi)$. Thus at such a point we find from (12) and substituting for $\rho$,

$$
\frac{\partial^{2} h}{\partial^{2} \theta}=\frac{\sin p \theta}{\sin ^{2} \theta}\{\sin p \theta \cos (p+1) \theta-p \sin \theta\}
$$

and so the second factor is negative. Thus $\partial^{2} h / \partial \theta^{2}<0$ only if $\sin p \theta>$ 0 , i.e. if $r$ is odd. Moreover at a local maximum we have using (13)

$$
h=\frac{\sin p \theta}{(p+1) \sin \theta} \text {. }
$$

Thus if $\theta \leqq \pi / 2$ we we find that except on the first branch $\theta>2 \pi / p$ and so 


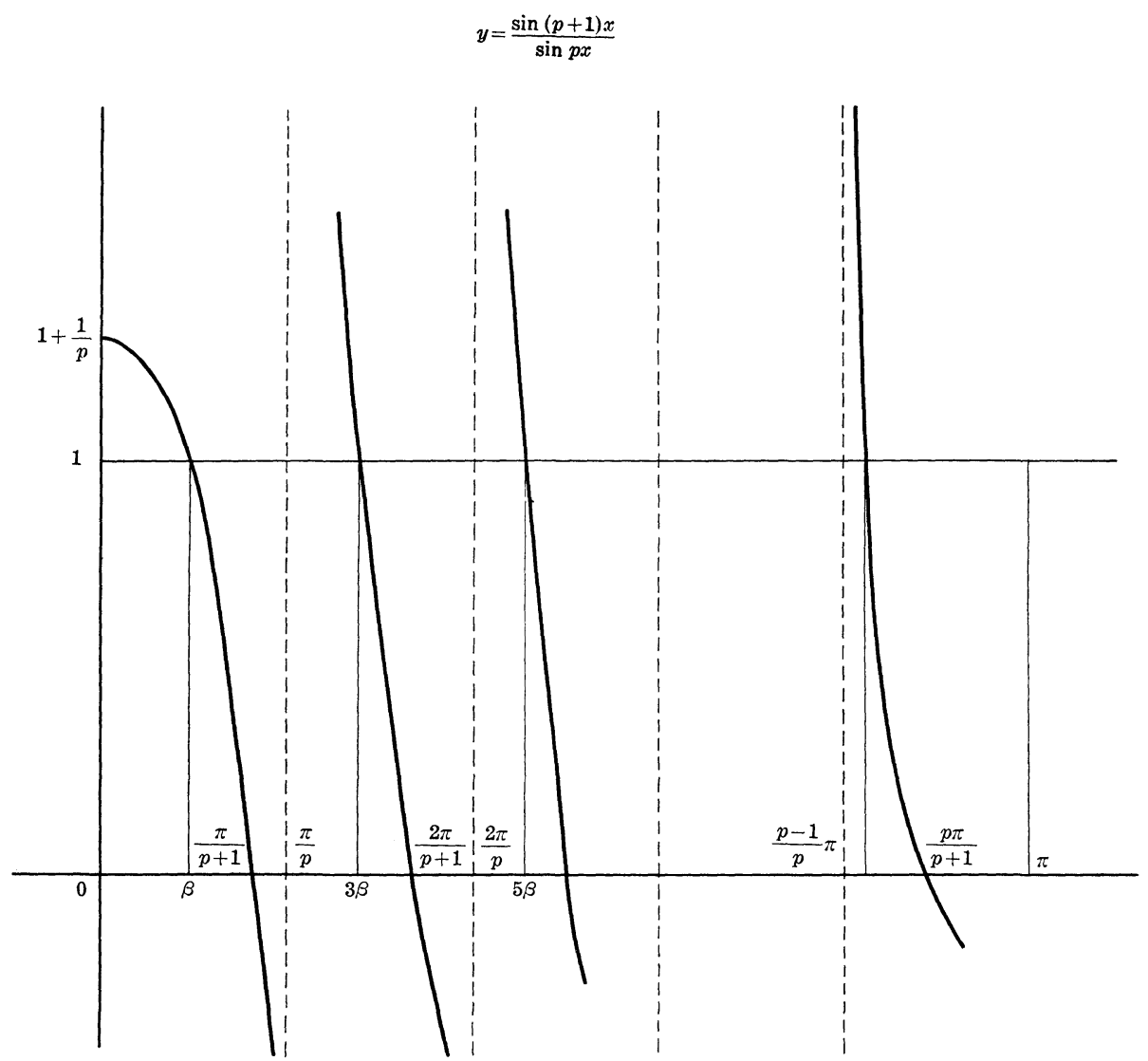

FIGURE 1

$$
\begin{aligned}
h & \leqq \frac{1}{(p+1) \sin \theta} \\
& \leqq \frac{\pi}{2(p+1) \theta} \\
& \leqq \frac{p}{4(p+1)}<\sigma_{p} .
\end{aligned}
$$

Similarly if $\theta \geqq \pi / 2$ we find that

$$
\pi-\theta \geqq \pi-\frac{\pi p}{p+1}=\frac{\pi}{p+1}
$$

and so

$$
\begin{aligned}
h & \leqq \frac{1}{(p+1) \sin \theta} \\
& \leqq \frac{1}{p+1} \operatorname{cosec} \frac{\pi}{p+1} \\
& \leqq \frac{1}{3} \operatorname{cosec} \frac{1}{3} \pi<\sigma_{p},
\end{aligned}
$$


since $x \operatorname{cosec} x$ increases over $(0, \pi / 2)$.

Thus we need only consider the first branch. Let $k(\rho)$ be the value taken by $h(z, p)$ with $\rho \in(0,1)$ and $\theta$ defined by $\rho \sin p \theta=$ $\sin (p+1) \theta, \theta \in(0, \pi /(p+1))$. Then

$$
\begin{aligned}
\frac{d k}{d \rho} & =\frac{\partial h}{\partial \rho}+\frac{\partial h}{\partial \theta} / \frac{d \rho}{d \theta}=\frac{\partial h}{\partial \rho} \\
& =-\frac{(p+1) k}{\rho}+\frac{1}{\rho} \frac{\sin p \theta}{\sin \theta} .
\end{aligned}
$$

Thus

$$
\begin{aligned}
\frac{d}{d \rho}\left\{\rho \frac{d k}{d \rho}+(p+1) k\right\} & =\frac{d}{d \theta}\left(\frac{\sin p \theta}{\sin \theta}\right) / \frac{d}{d \theta}\left(\frac{\sin (p+1) \theta}{\sin p \theta}\right) \\
& >0,
\end{aligned}
$$

since both $(\sin p \theta) /(\sin \theta)$ and $(\sin (p+1) \theta) /(\sin p \theta)$ decrease over $(0$, $\pi /(p+1))$. Therefore

$$
\frac{d}{d \rho}\left\{\rho^{p+2} \frac{d k}{d \rho}\right\}=\rho^{p+1} \frac{d}{d \rho}\left\{\rho \frac{d k}{d p}+(p+1) k\right\}>0,
$$

and so $\rho^{p+2} d k / d \rho$ increases. But as $\rho \rightarrow 0, \theta \rightarrow \pi /(p+1)$, and so using (17) we see that $\rho^{p+2} d k / d \rho \rightarrow 0$. Thus for $\rho>0, d k / d \rho>0$, whence $k$ increases over $(0,1)$. Thus for all such $z, h(z, p) \leqq k(1)=\sigma_{p}$ with equality if and only if $z=\exp i \pi /(2 p+1)$. This concludes the discussion of this case.

5. The case $\rho \geqq 1$. We find that

$$
\frac{\partial}{\partial \theta}\left\{\frac{1}{2} \log \left(1-2 \rho \cos \theta+\rho^{2}\right)+\rho \cos \theta\right\}=\frac{\rho^{2} \sin \theta(2 \cos \theta-\rho)}{1-2 \rho \cos \theta+\rho^{2}}
$$

and so if $\rho \geqq 2,1 / 2 \log \left(1-2 \rho \cos \theta+\rho^{2}\right)+\rho \cos \theta \leqq \log (\rho-1)+\rho$, whence for $\rho \geqq 2$,

$$
\begin{aligned}
g(z, p) & =\rho^{-p}\left\{\frac{1}{2} \log \left(1-2 \rho \cos \theta+\rho^{2}\right)+\sum_{1}^{p} \frac{\rho^{r}}{r} \cos r \theta\right\} \\
& \leqq \rho^{-p}\left\{\log (\rho-1)+\sum_{1}^{p} \frac{\rho^{r}}{r}\right\}=g(\rho, p) .
\end{aligned}
$$

Also $g(\rho, 2)$ is decreasing for $\rho>2$, for by $(10)$ we find that

$$
\frac{d g(\rho, 2)}{d \rho}=\frac{-2}{\rho^{3}} \log (\rho-1)-\frac{\rho-2}{\rho^{2}(\rho-1)} .
$$

But we now see from the definition of $g(z, p)$ that

$$
g(\rho, p+1)=\frac{1}{p+1}+\frac{1}{\rho} g(\rho, p)
$$


and so by induction we see that $g(\rho, p)$ decreases for $\rho \geqq 2$ for each $p \geqq 2$. Thus

$$
\text { for } \rho \geqq 2, p \geqq 2 g(z, p) \leqq g(2, p),
$$

with equality only for $z=2$.

Consider first the case $p=1$. If $\rho \leqq 2$, we find that for given $\rho, g$ is greatest when $2 \cos \theta=\rho$, or $g(z, 1) \leqq \rho / 2 \leqq 1$ for $\rho \leqq 2$. For $2 \leqq \rho$, we know that $g(z, 1) \leqq g(\rho, 1)=\rho^{-1} \log (\rho-1)+1$, and it is easily seen that this expression has precisely one turning value, and that a maximum, which occurs where $(\rho-1) \log (\rho-1)=\rho$ : This gives $\rho=4.5911$ and then $g(\rho, 1)=1.2785$. Thus $a_{1}=1.2785$.

Secondly, consider $p=2$. For $\rho \leqq 2$ we have

$$
\begin{aligned}
\rho^{2} g(z, 2) & =\frac{1}{2} \log \left(1-2 \rho \cos \theta+\rho^{2}\right)+\rho \cos \theta+\frac{1}{2} \rho^{2} \cos 2 \theta \\
& \leqq \frac{1}{2} \rho^{2}+\frac{1}{2} \rho^{2} \cos 2 \theta, \text { as before } \\
& \leqq \rho^{2},
\end{aligned}
$$

where equality occurs only if $\rho=2 \cos \theta$ and $\cos 2 \theta=1$ are satisfied simultaneously; this does occur and at the single point $z=2$. Thus $a_{2}=1$.

Finally we consider $p \geqq 3$, and then in view of (18) we need only consider the annulus $1 \leqq|z| \leqq 2$. At a local maximum, we obtain from (8), $\rho \sin p \theta=\sin (p+1) \theta$. In view of (9) $\theta=0$ arises only if $\rho \geqq 1+p^{-1}$, since otherwise $\partial^{2} g / \partial \theta^{2}$ is positive. $\theta=\pi$ can be dismissed, since by (10) a local maximum at such a point would give $g \leqq p^{-1} \leqq$ $1 / 3<\sigma_{p}$, by (16). Referring to the figure, we find therefore that we need to consider three cases

(a) $\theta=0$ for $\rho \geqq 1+p^{-1}$,

(b) $0<\theta \leqq \pi /(2 p+1)$ for $1 \leqq \rho \leqq 1+p^{-1}$,

(c) values of $\theta$ between $\pi / p$ and $\pi-\pi /(p+1)$.

As before the final case can be dismissed, since at such a local maximum we find from (10) that

$$
\begin{aligned}
g & =\frac{\sin (p+1) \theta}{p \sin \theta} \\
& \leqq p^{-1} \operatorname{cosec} \frac{\pi}{p+1} \\
& =\frac{p+1}{\pi p} \frac{\pi}{p+1} \operatorname{cosec} \frac{\pi}{p+1} \\
& \leqq \frac{p+1}{p} \frac{1}{4} \operatorname{cosec} \frac{1}{4} \pi \\
& \leqq \frac{1}{3} \operatorname{cosec} \frac{1}{4} \pi=\frac{1}{3} 2^{1 / 2}<\sigma_{p}, \text { in view of }(16) .
\end{aligned}
$$


Now in the second case, let $m(\rho)$ be the value taken by $g(z, p)$ when $\rho \sin p \theta=\sin (p+1) \theta$ and $0<\theta \leqq \pi /(2 p+1)$. Then using (8) and (10) we obtain similarly to (17),

$$
\frac{d m}{d \rho}=\frac{-p m}{\rho}+\frac{\sin p \theta}{\sin \theta}
$$

and so

$$
\begin{aligned}
\frac{d}{d \rho}\left\{\rho^{p+1} \frac{d m}{d \rho}\right\} & =\rho^{p} \frac{d}{d \rho}\left\{\rho \frac{d m}{d \rho}+p m\right\} \\
& =\rho^{p} \frac{d}{d \theta}\left\{\frac{\sin (p+1) \theta}{\sin \theta}\right\} / \frac{d}{d \theta}\left\{\frac{\sin (p+1) \theta}{\sin p \theta}\right\} \\
& >0, \text { as before. }
\end{aligned}
$$

Thus $\rho^{p+1} d m / d \rho$ increases as $\rho$ increases from 1 to $1+p^{-1}$. But using (19) we see that when $\rho=1$,

$$
\begin{aligned}
\frac{d m}{d \rho} & =-p \sigma_{p}+\sin \frac{p \pi}{2 p+1} / \sin \frac{\pi}{2 p+1} \\
& =-p \sigma_{p}+\frac{1}{2} \operatorname{cosec} \frac{\pi}{4 p+2} \\
& >-p \sigma_{p}+(2 p+1) / \pi \\
& >p\left(2 / \pi-\sigma_{p}\right)>0, \text { in view of }(16) .
\end{aligned}
$$

Thus $m(\rho)$ is an increasing function of $\rho$ as $\rho$ increases from 1 to $1+p^{-1}$, and in particular $g\left(1+p^{-1}, p\right) \geqq g(z, p)$ for $|z| \leqq 1+p^{-1}$. Thus we need only consider case (a).

Let

$$
\begin{aligned}
\Delta_{p} & =f\left(1+p^{-1}, p\right) \\
& =-\log p+\sum_{1}^{p} \frac{1}{r}\left(1+\frac{1}{p}\right)^{r} \\
& =-\log p+\sum_{1}^{p} \int_{0}^{1+p^{-1}} t^{r-1} d t \\
& =-\log p+\int_{0}^{1+p^{-1}} \frac{t^{p}-1}{t-1} d t .
\end{aligned}
$$




$$
\begin{aligned}
\Delta_{p+1}-\Delta_{p}= & -\log \frac{p+1}{p}+\int_{0}^{1+(p+1)^{-1}} \frac{t^{p+1}-t^{p}}{t-1} d t \\
& -\int_{1+(p+1)-1}^{1+p^{-1}} \frac{t^{p}-1}{t-1} d t \\
= & -\log \frac{p+1}{p}+\frac{1}{p+1}\left\{1+\frac{1}{p+1}\right\}^{p+1}+\log \frac{p+1}{p} \\
& -\int_{1+(p+1)-1}^{1+p^{-1}} \frac{t^{p}}{t-1} d t \\
= & \frac{1}{p+1}\left\{1+\frac{1}{p+1}\right\}^{p+1}-I, \text { say } .
\end{aligned}
$$

To estimate $I$ we observe that for $t>1+(p+1)^{-1}$,

$$
\frac{d}{d t}\left(\frac{t^{p+2}}{t-1}\right)=\frac{(p+1) t^{p+1}}{(t-1)^{2}}\left(t-1-\frac{1}{p+1}\right)>0,
$$

and so

$$
\begin{aligned}
I & =\int_{1+(p+1)-1}^{1+p^{-1}} \frac{t^{p+2}}{t-1} \frac{d t}{t^{2}} \\
& >(p+1)\left\{1+\frac{1}{p+1}\right\}^{p+2} \int_{1+(p+1)-1}^{1+p^{-1}} \frac{d t}{t^{2}} \\
& =(p+2)\left\{1+\frac{1}{p+1}\right\}^{p+1}\left\{\frac{p+1}{p+2}-\frac{p}{p+1}\right\} \\
& =\frac{1}{p+1}\left\{1+\frac{1}{p+1}\right\}^{p+1},
\end{aligned}
$$

and so

$$
\Delta_{p+1}<\Delta_{p}
$$

From (10) we see that if $\theta=0, \partial g / \partial \rho=-p g / \rho+(\rho-1)^{-1}$, and so it is easily verified that $\partial g / \partial \rho>0$ at $z=1+p^{-1}$, and that $\partial g / \partial \rho<$ 0 at $z=2$. Thus there exists at least one turning value of $g$ on the real axis between these two points. At such a point $g=\rho / p(\rho-1)$ and so

$$
\begin{aligned}
\frac{\partial^{2} g}{\partial \rho^{2}} & =-\frac{p}{\rho} \frac{\partial g}{\partial \rho}+\frac{p g}{\rho^{2}}-\frac{1}{(\rho-1)^{2}} \\
& =\frac{1}{\rho(\rho-1)}-\frac{1}{(\rho-1)^{2}}<0,
\end{aligned}
$$

and so there is exactly one such turning value, and that a maximum. Now let

$$
\mu(x, p)=g\left(1+x p^{-1}, p\right), x \geqq 1 .
$$


Then using (20) we find

$$
\begin{aligned}
\mu(x, p) & =\left(1+\frac{x}{p}\right)^{-p}\left\{\log \frac{x}{p}+\sum_{1}^{p} \frac{1}{r}\left(1+\frac{x}{p}\right)^{r}\right\} \\
= & \left(1+\frac{x}{p}\right)^{-p}\left\{\Delta_{p}+\log x+\sum_{1}^{p} \frac{1}{r}\left\{\left(1+\frac{x}{p}\right)^{r}-\left(1+\frac{1}{p}\right)^{r}\right\}\right\} \\
& =\left(1+\frac{x}{p}\right)^{-p}\left\{\Delta_{p}+\log x+\sum_{1}^{p} \int_{1+p^{-1}}^{1+x p^{-1}} t^{r-1} d t\right\} \\
& =\left(1+\frac{x}{p}\right)^{-p}\left\{\Delta_{p}+\log x+\int_{1+p^{-1}}^{1+x p^{-1}} \frac{t^{p}-1}{t-1} d t\right\} \\
& =\left(1+\frac{x}{p}\right)^{-p}\left\{\Delta_{p}+\int_{1}^{x}\left(1+\frac{s}{p}\right)^{p} \frac{d s}{s}\right\} \\
& =A_{p}(x)+B_{p}(x), \text { say. }
\end{aligned}
$$

Now, $(1+x / n)^{n}$ is an increasing sequence and by (21) $\Delta_{n}$ is decresing. Thus $A_{p+1}(x)<A_{p}(x)$. We shall show that $B_{p+1}(x) \leqq B_{p}(x)$ too. We find that for $s<x$,

$$
\begin{aligned}
\frac{\left(1+\frac{s}{p}\right)^{p}}{\left(1+\frac{x}{p}\right)^{p}} & \div \frac{\left(1+\frac{s}{p+1}\right)^{p+1}}{\left(1+\frac{x}{p+1}\right)^{p+1}}=\frac{(p+s)^{p}(p+1+x)^{p+1}}{(p+x)^{p}(p+1+s)^{p+1}} \\
& =\frac{p+1+x}{p+1+s}\left\{1+\frac{x-s}{p^{2}+p(x+s+1)+s+s x}\right\}^{-p} \\
& >\frac{p+1+x}{p+1+s}\left\{1-\frac{p(x-s)}{p^{2}+p(x+s+1)+s+s x}\right\} \\
& =\frac{p^{2}+p(2 s+1)+s+s x}{p^{2}+p(2 s+1)+s+s^{2}}>1,
\end{aligned}
$$

since $(1+\varepsilon)^{-p}>1-p \varepsilon$ for every positive $\varepsilon$. Thus

$$
\left(1+\frac{x}{p}\right)^{-p}\left(1+\frac{s}{p}\right)^{p}>\left(1+\frac{x}{p+1}\right)^{-p-1}\left(1+\frac{s}{p+1}\right)^{p+1}
$$

for $1 \leqq s<x$, and so $B_{p}(x) \geqq B_{p+1}(x)$.

We see therefore that

$$
g\left(1+\frac{x}{p+1}, p+1\right)<g\left(1+\frac{x}{p}, p\right) \leqq a_{p},
$$

and so $a_{p+1}<a_{p}$.

Also, since $\mu(x, p)>\mu(x, p+1)$, we see that

$$
\mu(x, p)>\mu(x)=\lim _{p \rightarrow \infty} \mu(x, p)=e^{-x}\left\{\Delta+\int_{1}^{x} s^{-1} e^{s} d s\right\},
$$


where $\Delta=\lim _{p \rightarrow \infty} \Delta_{p}$. Now from (20) we find that

$$
\begin{aligned}
\Delta_{p} & =-\log p+\sum_{1}^{p} \frac{1}{r}+\sum_{1}^{p} \frac{1}{r}\left\{\left(1+\frac{1}{p}\right)^{r}-1\right\} \\
& =-\log p+\sum_{1}^{p} \frac{1}{r}+\sum_{1}^{p} \int_{0}^{1} \frac{1}{p}\left(1+\frac{s}{p}\right)^{r-1} d s, \\
& =-\log p+\sum_{1}^{p} \frac{1}{r}+\int_{0}^{1}\left\{\left(1+\frac{s}{p}\right)^{p}-1\right\} \frac{d s}{s},
\end{aligned}
$$

and so

$$
\Delta=\gamma+\int_{0}^{1}\left(e^{s}-1\right) s^{-1} d s=1.895118
$$

Now

$$
\begin{gathered}
\mu^{\prime}(x)=-\mu(x)+x^{-1} \\
\mu^{\prime \prime}(x)=-\mu^{\prime}(x)-x^{-2},
\end{gathered}
$$

and so $\mu(x)$ has precisely one maximum, and at this point $\mu(x)=$ $x^{-1}$, with

$$
x^{-1} e^{x}=\Delta+\int_{1}^{x} s^{-1} e^{s} d s=\Delta+x^{-1} e^{x}-e+\int_{1}^{x} s^{-2} e^{s} d s
$$

or

$$
\int_{1}^{x} s^{-2} e^{s} d s=e-\Delta=0.823164,
$$

whence $x=1.3472$ and so $\mu_{\max }=0.7423$.

Thus we find that since $\mu(x, p)>\mu(x)$, we can always choose $x$ such that $\mu(x, p)>0.7423$, and so $a_{p}>0.7423$. Thus as $p$ increases from 2 to $\infty, a_{p}$ decreases from 1 to 0.7423 .

\section{REFERENCES}

1. E. Hille, Analytic Function Theory, Vol. II, Boston 1962. p. 195.

2. R. Redheffer, Problem 5628, Amer. Math. Monthly, 75 (1968).

Received May 11, 1970 and in revised form December 22, 1970.

Royal Holloway College, Englefield Green, Surrey, England 


\section{PACIFIC JOURNAL OF MATHEMATICS}

\section{EDITORS}

H. SAMELSON

Stanford University

Stanford, California 94305

C. R. Hовву

University of Washington

Seattle, Washington 98105
J. DugundJI

Department of Mathematics University of Southern California

Los Angeles, California 90007

RICHARD ARENS

University of California

Los Angeles, California 90024

\section{ASSOCIATE EDITORS}

E. F. BECKENBACH

B. H. NeumanN

F. WOLF

K. YoSHIDA

\section{SUPPORTING INSTITUTIONS}

UNIVERSITY OF BRITISH COLUMBIA

CALIFORNIA INSTITUTE OF TECHNOLOGY

UNIVERSITY OF CALIFORNIA

MONTANA STATE UNIVERSITY

UNIVERSITY OF NEVADA

NEW MEXICO STATE UNIVERSITY

OREGON STATE UNIVERSITY

UNIVERSITY OF OREGON

OSAKA UNIVERSITY
UNIVERSITY OF SOUTHERN CALIFORNIA

STANFORD UNIVERSITY

UNIVERSITY OF TOKYO

UNIVERSITY OF UTAH

WASHINGTON STATE UNIVERSITY

UNIVERSITY OF WASHINGTON

AMERICAN MATHEMATICAL SOCIETY

NAVAL WEAPONS CENTER

Printed in Japan by International Academic Printing Co., Ltd., Tokyo, Japan 


\section{Pacific Journal of Mathematics}

\section{Vol. 44, No. $1 \quad$ May, 1973}

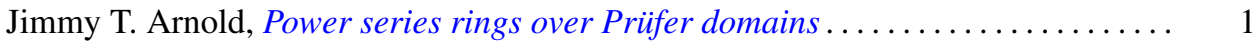

Maynard G. Arsove, On the behavior of Pincherle basis functions . . . . . . . . . 13

Jan William Auer, Fiber integration in smooth bundles ................. 33

George Bachman, Edward Beckenstein and Lawrence Narici, Function algebras

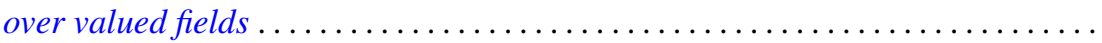

Gerald A. Beer, The index of convexity and the visibility function . . . . . . . . . . .

James Robert Boone, A note on mesocompact and sequentially mesocompact

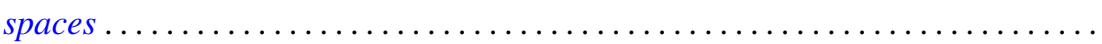

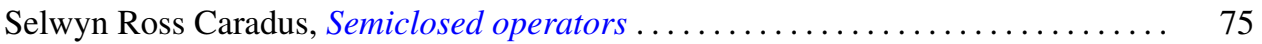

John H. E. Cohn, Two primary factor inequalities . . . . . . . . . . . . . . . 81

Mani Gagrat and Somashekhar Amrith Naimpally, Proximity approach to

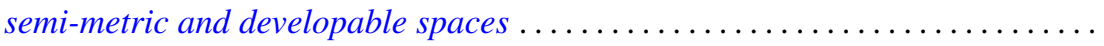

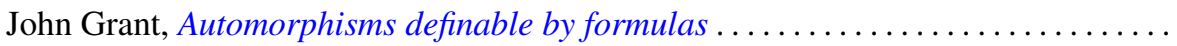

Walter Kurt Hayman, Differential inequalities and local valency ..............

Wolfgang H. Heil, Testing 3-manifolds for projective planes . . . . . . . . . . . . .

107

Melvin Hochster and Louis Jackson Ratliff, Jr., Five theorems on Macaulay

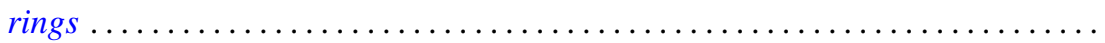

Thomas Benton Hoover, Operator algebras with reducing invariant subspaces ....

James Edgar Keesling, Topological groups whose underlying spaces are separable

Fréchet manifolds...

Frank Leroy Knowles, Idempotents in the boundary of a Lie group . .

191

George Edward Lang, The evaluation map and EHP sequences ...

201

Everette Lee May, Jr, Localizing the spectrum . . . . . . . . . . . .

211

Frank Belsley Miles, Existence of special $K$-sets in certain locally compact abelian groups.

Susan Montgomery, A generalization of a theorem of Jacobson. II . .

T. S. Motzkin and J. L. Walsh, Equilibrium of inverse-distance forces in

three-dimensions.

Arunava Mukherjea and Nicolas A. Tserpes, Invariant measures and the converse

of Haar's theorem on semitopological semigroups .

James Waring Noonan, On close-to-convex functions of order $\beta$

Donald Steven Passman, The Jacobian of a growth transformation

Dean Blackburn Priest, A mean Stieltjes type integral ........ .

Joe Bill Rhodes, Decomposition of semilattices with applications to topological

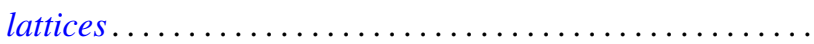

Claus M. Ringel, Socle conditions for $\mathrm{QF}-1$ rings ..........

Richard Rochberg, Linear maps of the disk algebra

Roy W. Ryden, Groups of arithmetic functions under Dirichlet convolution . .

Michael J. Sharpe, A class of operators on excessive functions

Erling Stormer, Automorphisms and equivalence in von Neumann algebras ..

Philip C. Tonne, Matrix representations for linear transformations on series

analytic in the unit disc. 